\title{
DESIGN OF A LOAD FOLLOWING CONTROLLER FOR APR+ NUCLEAR PLANTS
}

\author{
SIM WON LEE ${ }^{1}$, JAE HWAN KIM ${ }^{1}$, MAN GYUN NA ${ }^{1 *}$, DONG SU KIM ${ }^{2}$, KEUK JONG YU ${ }^{3}$, and HAN GON KIM ${ }^{3}$ \\ ${ }^{1}$ Department of Nuclear Engineering, Chosun University \\ 375 Seosuk-dong, Dong-gu, Gwangju 501-759, Republic of Korea \\ ${ }^{2}$ Korea Atomic Energy Research Institute \\ Dukjin-dong, Yuseong-gu, Daejon 305-600, Republic of Korea \\ ${ }^{3}$ Central Research Institute, KHNP \\ 25-1 jang-dong, Deajeon 305-343, Republic of Korea \\ *Corresponding author. E-mail : magyna@chosun.ac.kr
}

Invited September14, 2011

Received November 03, 2011

Accepted for Publication February 21, 2012

A load-following operation in APR+ nuclear plants is necessary to reduce the need to adjust the boric acid concentration and to efficiently control the control rods for flexible operation. In particular, a disproportion in the axial flux distribution, which is normally caused by a load-following operation in a reactor core, causes xenon oscillation because the absorption cross-section of xenon is extremely large and its effects in a reactor are delayed by the iodine precursor. A model predictive control (MPC) method was used to design an automatic load-following controller for the integrated thermal power level and axial shape index (ASI) control for APR+ nuclear plants. Some tracking controllers employ the current tracking command only. On the other hand, the MPC can achieve better tracking performance because it considers future commands in addition to the current tracking command. The basic concept of the MPC is to solve an optimization problem for generating finite future control inputs at the current time and to implement as the current control input only the first control input among the solutions of the finite time steps. At the next time step, the procedure to solve the optimization problem is then repeated. The support vector regression (SVR) model that is used widely for function approximation problems is used to predict the future outputs based on previous inputs and outputs. In addition, a genetic algorithm is employed to minimize the objective function of a MPC control algorithm with multiple constraints. The power level and ASI are controlled by regulating the control banks and part-strength control banks together with an automatic adjustment of the boric acid concentration. The 3-dimensional MASTER code, which models APR + nuclear plants, is interfaced to the proposed controller to confirm the performance of the controlling reactor power level and ASI. Numerical simulations showed that the proposed controller exhibits very fast tracking responses.

KEYWORDS : SVR Model, Genetic Algorithm, Model Predictive Control, Nuclear Reactor Power and ASI Control

\section{INTRODUCTION}

Nuclear power plant characteristics vary according to the operating power levels. Ageing effects in plant performance and changes in the nuclear core reactivity with fuel burnup generally degrade the system performance, which means that power plants are quite complex, nonlinear, time-varying and constrained systems. Advanced power and ASI tracking control of nuclear reactors has not been accepted mainly due to the safety concerns of imprecise knowledge of the time-varying parameters, nonlinearity and modeling uncertainty. On the other hand, rapid and smooth power maneuvering has its benefits in terms of the economical and safe operation of reactors. Nuclear reactor power and ASI should be controlled properly to establish good operation performance and maximize the thermal efficiency of nuclear power plants. Therefore, we used a model predictive control method to design an automatic controller for thermal power and ASI control in APR+ nuclear plants.

This study employs model predictive control methodology that has attracted considerable attention as a powerful tool for the control of industrial process systems [1]-[8] and combines the support vector regression (SVR) model that is used widely in a range of engineering fields. The basic concept of model predictive control is to solve an optimization problem for a finite future at the current time, and once a future input trajectory has been chosen, 
only the first element of that trajectory is used as input to the plant. The SVR model is used to predict the future output required in an optimization problem. That is, the behavior of the process over a prediction horizon at the present time is considered and the process output to changes in the manipulated variable is predicted using the SVR model.

The moves of the manipulated variables are selected so that the predicted output has certain desirable characteristics. On the other hand, only the first computed change in the manipulated variable is implemented and the procedure is repeated at each subsequent instant. This method has many advantages over conventional infinite horizon control because it is possible to handle input and output constraints in a systematic manner during the design and implementation of the control. In particular, it is a suitable control strategy for nonlinear time varying systems because of the model predictive concept. Recently, the problem of controlling uncertain dynamical systems has been of considerable interest to control engineers. This paper deals systematically with the multiple objectives related to control input using a genetic algorithm and predicts the outputs using the SVR method to improve the performance.

Based on an SVR reactor model consisting of the control rod position, the past nuclear reactor power and ASI, the future nuclear reactor power and ASI were predicted using the SVR model. The objective function for the model predictive control was minimized using a genetic algorithm. A 3-dimensional nuclear reactor analysis code that was developed by Korea Atomic Energy Research Institute (KAERI) was used to confirm the proposed controller for nuclear reactor power.

\section{CONVENTIONAL POWER CONTROL SYSTEM FOR PWRS}

Most of the existing PWRs have two types of control mechanisms, control rods and chemical shim. In many power reactors, the control rod drives are interconnected electrically so that several control rods can move simultaneously in response to a signal from the reactor operator. Many power reactors can be controlled in part by varying the boric acid $\left(\mathrm{H}_{3} \mathrm{BO}_{3}\right)$ concentration in the reactor coolant because boron absorbs neutrons quite well, which is called chemical shim control. The control rods provide reactivity control for rapid shutdown and for compensating for reactivity changes due to temperature changes that accompany the changes in power. The chemical shim is used to keep the reactor critical during xenon transients which have a very slow response, and to compensate for fuel depletion and fission product buildup over the life of the reactor core.

The conventional reactor regulating system normally controls the average temperature of the reactor core according to a reference temperature that is proportional to the turbine load to maximize the plant thermal efficiency. The conventional control method consists of a temperature deviation channel (the difference between the reference average coolant temperature and the average coolant temperature) and a power mismatch channel (the difference between the turbine load and the nuclear power). The conventional controller generates the insertion or withdrawal speed of the reactor control rods using the error signals obtained by compensating for and filtering these two channels. The control rod drive mechanism control system moves the control rod assembly groups according to the signals received. This conventional method has the advantages of easy implementation and well-proven technology. On the other hand, the techniques for the optimal power control of nuclear reactors have been studied extensively over the past two decades to optimize the reactor power control performance [9]-[12]. However, it is very difficult to design optimized controllers for nuclear systems because of the variations in nuclear system parameters and modeling uncertainties. Therefore, in this study, the MPC method was used to design an automatic controller for nuclear systems in APR+ nuclear plants.

\section{MODEL PREDICTIVE CONTROL}

In most nuclear power plants, the reactor regulating system (RRS) controls the reactor power (being operated in its rated power in the case of no particular problem and no load-following) with a manual adjustment of the boric acid concentration. The RRS employs conventional filters and compensators, which are difficult to optimize. The objective of the present study was to develop a new controller to replace the conventional RRS. Each bank of the control rod banks is distributed evenly in four radial quadrants of the reactor core to accomplish a symmetrical radial distribution. On the other hand, the control rods must move frequently if the plant is operated in a loadfollowing mode, which induces axial xenon oscillations. Power distribution control becomes increasingly important. This study focused on designing an excellent power level and ASI controller by introducing new methodologies, a model predictive control method, SVR and genetic algorithm. Nuclear reactor power and the ASI controller in the APR+ combines the model predictive control and SVR model. The model predictive control method aims to solve an optimization problem for a finite future at the current time and to implement the first optimal control input as the current control input. The procedure is then repeated at each subsequent time step. Fig. 1 shows the basic concept of the model predictive control [3]. For any assumed set of present and future control moves, the future behavior of the process outputs can be predicted over a prediction horizon, $N$, and the $M$ present and future control moves $(M \leq N)$ are calculated to minimize the quadratic objective function. Although $M$ control moves are 
calculated, only the first control move is implemented. At the next time step, new values of the measured output are obtained, the control horizon shifts forward by a single step, and the same calculations are repeated.

The purpose of taking new measurements at each time step is to compensate for any unmeasured disturbances and model inaccuracies, both of which cause the measured system output to differ from the one predicted by the model. At every time point, model predictive control requires a non-linear solution to an optimization problem to calculate the optimal control inputs over a fixed number of future time points, which are known as the time horizon. The basic idea of model predictive control is to calculate a sequence of future control signals in such a way that it minimizes the multistage cost function defined over a prediction horizon. In addition, to achieve rapid responses and prevent excessive control effort, the associated performance index for deriving an optimal control input is represented by the following quadratic function:

$$
J=\frac{1}{2} \sum_{k=1}^{N} Q[\hat{\mathbf{y}}(t+k \mid t)-\mathbf{w}(t+k)]^{2}+\frac{1}{2} \sum_{k=1}^{M} R[\Delta \mathbf{u}(t+k-1)]^{2}
$$

subject to constraints

$$
\left\{\begin{array}{c}
\Delta \mathbf{u}(t+k-1)=0 \text { for } k>M, \\
\mathbf{u}_{\min } \leq \mathbf{u}(t) \leq \mathbf{u}_{\max }, \\
-d \mathbf{u}_{\max } \leq \Delta \mathbf{u}(t) \leq d \mathbf{u}_{\max } .
\end{array}\right.
$$

where $Q$ and $R$ weight the reactor power, and the ASI (system output) error and control rod position (control input) change between neighboring time steps at certain future time intervals, respectively, and $\mathbf{w}$ is a setpoint (desired reactor power and ASI) or reference sequence for the output signal. $\hat{\mathbf{y}}(t+k \mid t)$ is the optimum $k$-stepahead prediction of the system outputs of the reactor power $\hat{y}_{1}(t+k \mid t)$ and ASI $\hat{y}_{2}(t+k \mid t)$ based on data up to time $t$, i.e., the expected output at time $t$ if the past input and output and future control sequence are known. $N$ and $M$ are called the prediction horizon and control horizon, respectively. The prediction horizon represents the limit of the instant desired for the output to follow the reference sequence. The constraint, $\Delta \mathbf{u}(t+k-1)=0$ for $k>M$, means there is no change in the control signals after a certain interval $M>N$, which is the control horizon concept. $\mathbf{u}_{\min }$ and $\mathbf{u}_{\max }$ are the minimum and maximum inputs, respectively, and $\Delta \mathbf{u}_{\max }$ is the maximum allowable control move per time step. To obtain the control inputs, the predicted outputs first need to be calculated as a function of previous inputs and outputs as well as future control signals, and the output prediction employs the SVR model. The SVR model is used widely for function approximation problems and has powerful capability. Therefore, we used the SVR model to predict the future output based on previous inputs and outputs, which will be described in the following section.

\subsection{0utput Prediction}

In this study, a SVR model was used to predict the future output. The inputs and outputs of the SVR model are real-valued variables. Therefore, SVR model searches for the network weights of an artificial neural network with

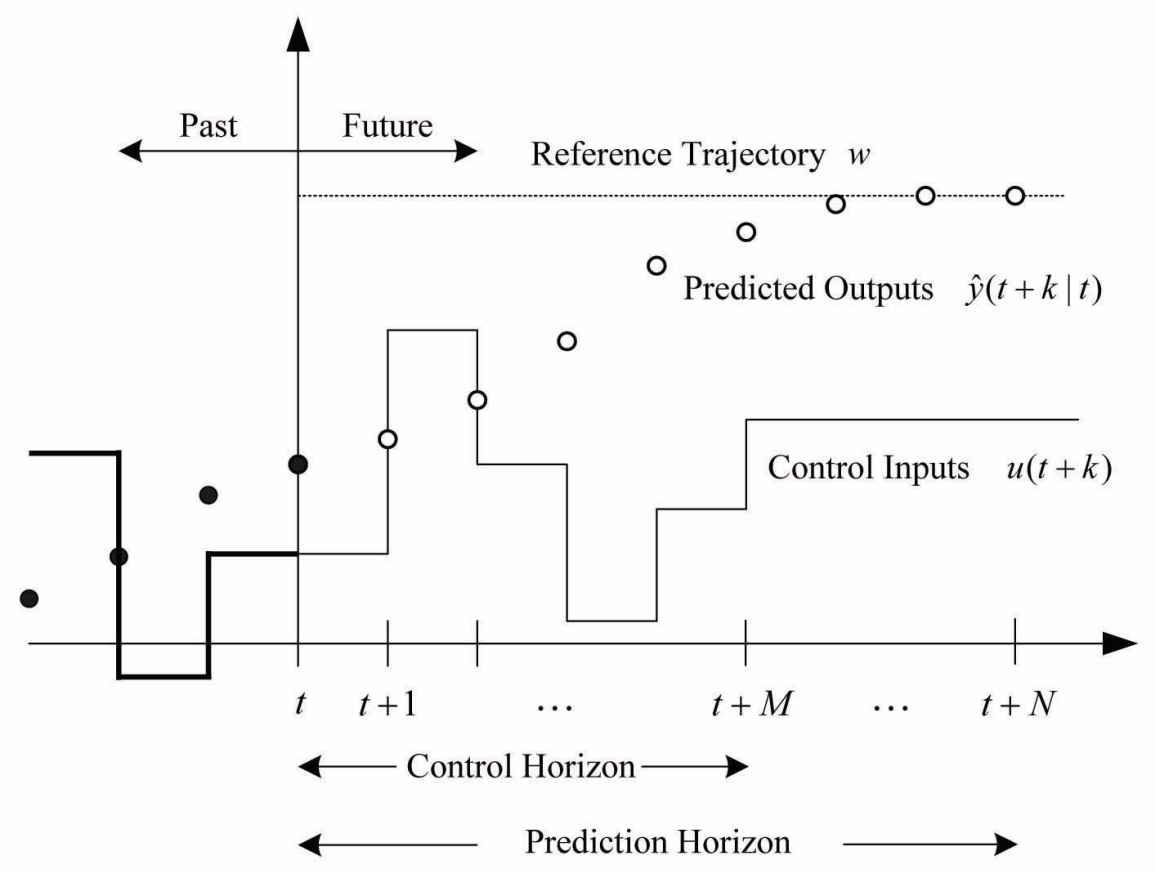

Fig. 1. MPC Concept. 
a kernel function by solving a non-convex unconstrained minimization problem. The SVR model employs the hypothesis space of linear functions in multi-dimensional feature space. This model is trained with a learning algorithm that has its origin in the theoretical foundations of statistical learning theory and structural risk minimization (SRM). Although both data modeling methods, NNs and SVR, show comparable results on the most popular benchmark problems, an attractive and promising area of research [13] is made by applying the theoretical status of SVR.

Fig. 2 shows the SRM principle [14]. The bound on the risk is the sum of the empirical risk and of the confidence interval. Although empirical risk minimization (ERM) methods minimize only the empirical risk at any cost, SRM methods determine the function $f^{*}$ that gives the smallest bound on the risk $R\left(f^{*}\right)$ for a given data set. In Fig. 2, the empirical risk decreases with an increasing capacity (with the index of the element of the structure, $d_{k}$ ), while the confidence interval increases. The smallest bound of the risk is achieved on some appropriate element of the structure. The difference in the risk minimization scheme causes better generalization in the SVR model than ANNs [14]. The SVR model can be applied well to regression and classification problems. This paper solves a typical regression problem with an approximation of an unknown function, which can be expressed as a linear expansion of basis functions.

The basic concept of the SVR is to map the original input data $\mathbf{x}$ nonlinearly into high dimensional feature space

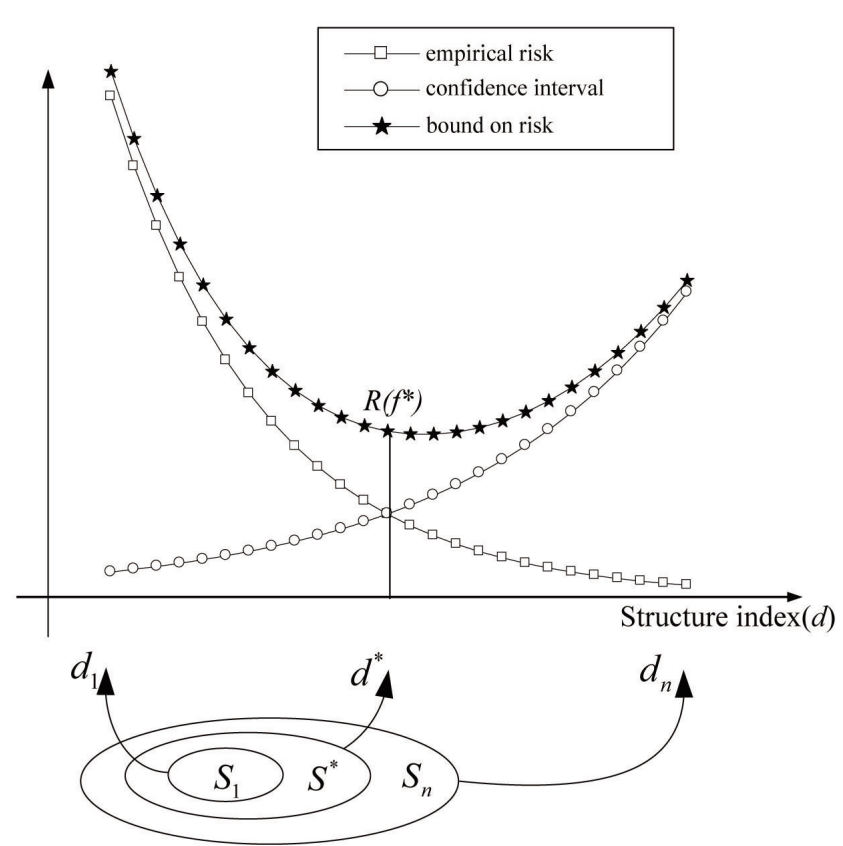

Fig. 2. Graphical Description of the SRM Principle. $\varphi$. The unknown function is solved by determining the coefficients of the basis function of the linear expansion. Therefore, for a given data set, $\left\{\left(\mathbf{x}_{k}, y_{k}\right)\right\}_{k=1}^{N} \in R^{m} \times R$, where $\mathbf{x}_{k}$ is the input vector to an SVR model, $y_{k}$ is the actual output value, $N$ is the total number of data and $m$ is the number of input signals. The support vector approximation can be expanded as follows:

$$
y=f(\mathbf{x})=\sum_{k=1}^{N} \omega_{k} \phi_{k}(\mathbf{x})=\boldsymbol{\omega}^{T} \boldsymbol{\varphi}(\mathbf{x})+b .
$$

The function $\phi_{k}(\mathbf{x})$ is called the feature and the parameters $\omega$ and $b$ are the support vector weight and bias, which are calculated by minimizing the following regularized risk function [14]:

$$
R(\boldsymbol{\omega})=\frac{1}{2} \boldsymbol{\omega}^{T} \boldsymbol{\omega}+\frac{\lambda}{h} \sum_{k=1}^{N}\left|y_{k}-f(\mathbf{x})\right|_{\varepsilon}^{h},
$$

where $h=1$ for the linear $\varepsilon$-insensitive loss function and $h=2$ for the quadratic $\varepsilon$-insensitive loss function. In this study, the linear $\varepsilon$-insensitive loss function $h=1$ was used. The linear $\varepsilon$-insensitive loss function is defined as follows [14]:

$$
\left|y_{k}-f(\mathbf{x})\right|_{\varepsilon}=\left\{\begin{array}{ll}
0 & \text { if }\left|y_{k}-f(\mathbf{x})\right|<\varepsilon \\
\left|y_{k}-f(\mathbf{x})\right|-\varepsilon & \text { otherwise }
\end{array} .\right.
$$

The first term of Eq. (3) stands for the complexity of the SVR model. The parameters, $\lambda$ and $\varepsilon$, are defined by the user. The loss is equal to zero if the difference between the estimated value and observed value is less than $\varepsilon$. For all other estimated points outside the error level, $\varepsilon$, the loss is equal to the magnitude of the difference between the estimated value and $\varepsilon$ (see Fig. 3). Minimizing the regularized risk function is equivalent to minimizing the following constrained risk function:

$$
R\left(\boldsymbol{\omega}, \xi, \xi^{*}\right)=\frac{1}{2} \boldsymbol{\omega}^{T} \boldsymbol{\omega}+\frac{\lambda}{h} \sum_{k=1}^{N}\left(\xi_{k}^{h}+\xi_{k}^{* h}\right)
$$

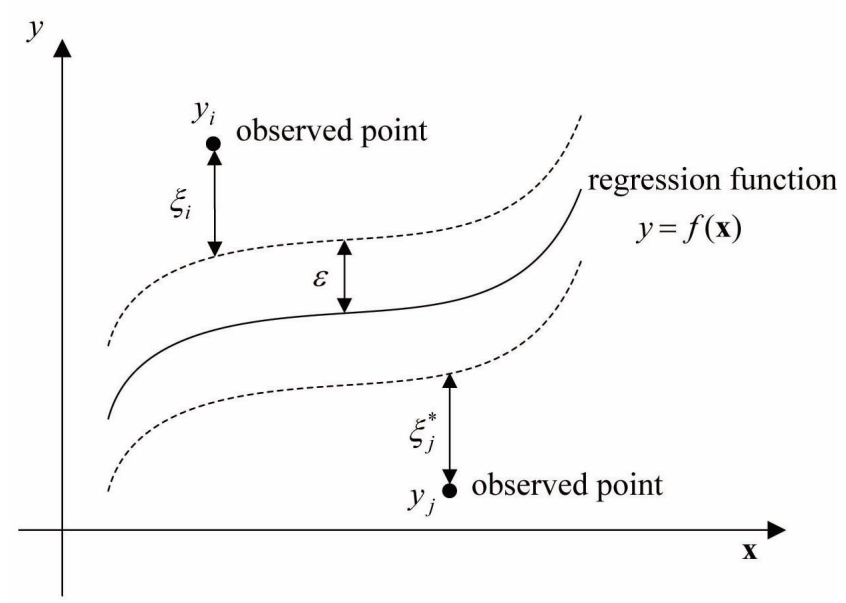

Fig. 3. Insensitive $\varepsilon$-tube for the SVR Model. 
subject to the constraints

$$
\begin{cases}y_{k}-\boldsymbol{\omega}^{T} \boldsymbol{\varphi}(\mathbf{x})-b \leq \varepsilon+\xi_{k}, & k=1,2, \cdots, N \\ \boldsymbol{\omega}^{T} \boldsymbol{\varphi}(\mathbf{x})+b-y_{k} \leq \varepsilon+\xi_{k}^{*}, & k=1,2, \cdots, N \\ \xi_{k}, \xi_{k}^{*} \geq 0, & k=1,2, \cdots, N\end{cases}
$$

where the constant $\lambda$ determines the trade-off between the complexity of $f(\mathbf{x})$ and the limit where deviations greater than $\varepsilon$ are tolerated. Parameters $\xi$ and $\xi^{*}$ are the slack (positive) variables that represent the upper and lower constraints on the system output, respectively.

The constrained optimization problem can be solved by applying the Lagrange multiplier technique to Eqs. (5) and (6). The regression function of Eq. (2) is expressed as follows:

$$
y=f(\mathbf{x})=\sum_{k=1}^{N} \gamma_{k} \boldsymbol{\varphi}^{T}\left(\mathbf{x}_{k}\right) \boldsymbol{\varphi}(\mathbf{x})+b=\sum_{k=1}^{N} \gamma_{k} K\left(\mathbf{x}, \mathbf{x}_{k}\right)+b,
$$

where $\gamma_{k}=\left(\alpha_{k}-\alpha_{k}^{*}\right)$ and $K\left(\mathbf{x}, \mathbf{x}_{k}\right)=\varphi^{T}\left(\mathbf{x}_{k}\right) \varphi(\mathbf{x})$ is known as the kernel function. Coefficients $\gamma_{k}$ are nonzero only for a subset of training data. The training data points, which correspond to non-zero $\gamma_{k}$, are known as support vectors (SVs) and have an approximation error $\geq \varepsilon$. In this study, the radial basis function can be described as follows:

$$
K\left(\mathbf{x}, \mathbf{x}_{k}\right)=\exp \left(-\frac{\left(\mathbf{x}-\mathbf{x}_{k}\right)^{T}\left(\mathbf{x}-\mathbf{x}_{k}\right)}{2 \sigma^{2}}\right) .
$$

The kernel function parameter $\sigma$ determines the sharpness of the radial basis kernel function. The bias $b$ is calculated as follows [15]:

$$
b=-\frac{1}{2} \sum_{k=1}^{N} \gamma_{k}\left(K\left(\mathbf{x}_{r}, \mathbf{x}_{k}\right)+K\left(\mathbf{x}_{s}, \mathbf{x}_{k}\right)\right),
$$

where $\mathbf{x}_{r}$ and $\mathbf{x}_{s}$ are SVs, which are data points outside the $\varepsilon$-insensitivity zone (refer to Fig. 3).

The two important design parameters for an SVR model are the insensitivity zone $\varepsilon$ and the regularization parameter $\lambda$. The parameter $\varepsilon$ determines the size of the insensitivity zone and controls the number of SVs. The increase in insensitivity zone $\varepsilon$ reduces the requirements for approximation accuracy and allows a decrease in the number of SVs, leading to data compression. In addition, an increase in the insensitivity zone $\varepsilon$ has a filtering effect on highly noisy data. The increase in regularization parameter $\lambda$ reduces the larger errors, which leads to a decrease in the approximation error. This can also be achieved by increasing the weight vector norm but an increase in the weight vector norm decreases the good generalization capability of the SVR model.

\subsection{Objective Function Optimization by a Genetic Algorithm}

The objective function of Eq. (1) can be solved using linear matrix inequality (LMI) techniques. In this study, a genetic algorithm was employed to minimize the objective function with multiple objectives. The genetic algorithm is suitable for solving multiple objective functions. Compared to conventional optimization methods, which move from one point to another, genetic algorithms begin from many points simultaneously by climbing many peaks in parallel. Accordingly, genetic algorithms are less susceptible to being stuck at local minima than conventional search methods [16]-[17].

In the genetic algorithm, the term chromosome refers to a candidate solution that minimizes a cost function. As the generation proceeds, populations of chromosomes are altered iteratively by biological mechanisms inspired by natural evolution, such as selection, crossover and mutation. The genetic algorithms require a fitness function that assigns a score to each chromosome (candidate solution) in the current population, and maximizes the fitness function value. The fitness function evaluates the extent to which each candidate solution is suitable for the specified objectives. The genetic algorithm begins with an initial population of chromosomes, which represent the possible solutions of the optimization problem. The fitness function is calculated for each chromosome. New generations are produced by the genetic operators known as selection, crossover and mutation. The algorithm stops after the maximum allowed time has elapsed.

In the following description [18]-[19], a chromosome will be represented by $s_{g}$, of which elements consist of present and future control inputs and will have the following structure:

$$
s_{g}=\left[\begin{array}{llll}
u_{g}(t) & u_{g}(t+1) & \cdots & u_{g}(t+M-1)
\end{array}\right], g=1, \cdots, G,
$$

where $t$ indicates the current time. Assuming the number of chromosomes, $G$, the crossover probability, $P_{c}$, and mutation probability, $P_{m}$, the algorithm proceeds according to the following steps:

Step 1 (initial population): Set the number of iterations iter $=1$. Generate an initial population consisting of $G$ chromosomes of Eq. (10). The values are allocated randomly but should satisfy both the input and input move constraints of Eq. (1). For this purpose, the following simple procedure was used:

(a) Read the measured value of the input variable at the previous time point $t-1$, which has already been implemented.

(b) Select the current input value using the following equations:

$$
\begin{gathered}
u_{g}(t)=u(t-1)+r \cdot \Delta u_{\max } . \\
\text { If } u_{g}(t) \geq u_{\max }, \text { set } u_{g}(t)=u_{\max } . \\
\text { If } u_{g}(t) \leq u_{\min }, \text { set } u_{g}(t)=u_{\min } .
\end{gathered}
$$

(c) Select the remaining input moves using the following 
equations:

$$
\begin{gathered}
u_{g}(t+k)=u_{g}(t+k-1)+r \cdot \Delta u_{\max }, 1 \leq k \leq M-1 . \\
\text { If } u_{g}(t+k) \geq u_{\max } \text {, set } u_{g}(t+k)=u_{\max }, 1 \leq k \leq M-1 . \\
\text { If } u_{g}(t+k) \leq u_{\min } \text {, set } u_{g}(t+k)=u_{\min }, 1 \leq k \leq M-1 .
\end{gathered}
$$

In the above equations, $r$ is a random number between -1 and 1 . A new random number $r$ is generated at each time when Eq. (11) or Eq. (14) is used.

Step 2 (fitness function evaluation): Evaluate the objective function of Eq. (1) for all chosen chromosomes. Invert the objective function values and determine the total fitness of the population as follows:

$$
F=\sum_{g=1}^{G} \frac{1}{J_{g}(t)},
$$

where $J_{g}(t)$ is the objective function value for the $g$-th chromosome and the inversion of $J_{g}(t)$ is a fitness value of the $g$-th chromosome. Calculate the normalized fitness value of each chromosome, which means selecting the probability $p_{g}$ calculated by

$$
p_{g}=\frac{1 / J_{g}(t)}{F}, g=1, \cdots, G .
$$

Step 3 (selection operation): Calculate the cumulative probability $q_{g}$ for each chromosome using the following equation:

$$
q_{g}=\sum_{j=1}^{g} p_{j}, g=1, \cdots, G .
$$

For $g=1, \ldots, G$, generate a random number, $r$, between 0 and 1. Select the chromosome for which $q_{g-1} \leq r \leq q_{g}$. At this point of the algorithm a new population of chromosomes has been generated. The chromosomes with a high fitness value have more chance to be selected.

Step 4 (crossover operation): For each chromosome, $s_{g}$, generate a random number $r$ between 0 and 1 . This particular chromosome will undergo a crossover process if $r<p_{c}$, otherwise it will remain unchanged. Mate the selected chromosomes and for each selected pair, generate a random integer number $z$ between 0 and $M-1$. The crossing point is the position indicated by the random number. Two new chromosomes are produced by interchanging all the members of the parents following the crossing point. Graphically, the crossover operation can be represented as shown below, assuming that the crossover operation is applied to the parent chromosomes, $s_{g}$ and $s_{g+1}$ :

$$
\begin{aligned}
& s_{g}=\left[\begin{array}{lllllll}
u_{g}(t) & u_{g}(t+1) & \cdots & u_{g}(t+z-1) & \mid u_{g}(t+z) & \cdots & u_{g}(t+M-1)
\end{array}\right]
\end{aligned}
$$

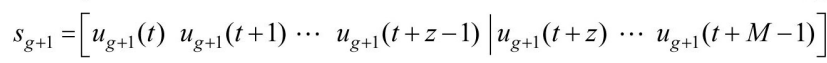

$$
\begin{aligned}
& \Downarrow \text { crossover operation } \\
& s_{g}^{n e w}=\left[\begin{array}{lllllll}
u_{g}(t) & u_{g}(t+1) & \cdots & u_{g}(t+z-1) & u_{g+1}(t+z) & \cdots & u_{g+1}(t+M-1)
\end{array}\right]
\end{aligned}
$$

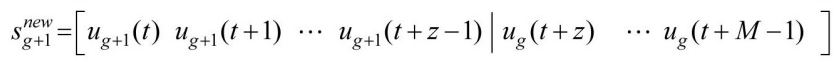

The above operation might produce infeasible offspring if the input values at the cross point do not satisfy the input move constraints. This situation is avoided by the following correction mechanism for an input variable, which modifies the input parameters after the cross position to satisfy the input move constraints. At first, for one of the chromosomes produced, $s_{g}^{\text {new }}$,

$$
\text { if } u_{g+1}(t+z)-u_{g}(t+z-1)>\Delta u_{\max },
$$

then

$$
\begin{aligned}
& \qquad \begin{array}{c}
\Delta=u_{g+1}(t+z)-u_{g}(t+z-1)-\Delta u_{\max }, \\
u_{g+1}(t+z+k)=u_{g+1}(t+z+k)-\Delta, 0 \leq k \leq M-1-z .
\end{array} \\
& \text { if } u_{g+1}(t+z)-u_{g}(t+z-1)<-\Delta u_{\max }, \\
& \text { then } \quad \Delta=u_{g}(t+z-1)-u_{g+1}(t+z)-\Delta u_{\max }, \\
& u_{g+1}(t+z+k)=u_{g+1}(t+z+k)+\Delta, \quad 0 \leq k \leq M-1-z .
\end{aligned}
$$

A similar set can be written for chromosome $s_{g+1}^{\text {new }}$.

Step 5 (mutation operation): For every member of each chromosome $s_{g}, u_{g}(t+k)$, generate a random number $r$ between 0 and 1 . If $r<p_{m}$, this particular member of the chromosome will undergo the process of mutation, otherwise it will remain unchanged. For the selected members define the upper and lower bounds as follows:

$$
\begin{gathered}
\text { if } k=0 \\
b_{u}=\min \left(\Delta u_{\max }+u(t-1), \Delta u_{\max }+u_{g}(t+k+1), u_{\max }\right) \\
b_{l}=\max \left(-\Delta u_{\max }+u(t-1),-\Delta u_{\max }+u_{g}(t+k+1), u_{\min }\right) \\
\text { if } 0<k<M-1 \\
b_{u}=\min \left(\Delta u_{\max }+u_{g}(t+k-1), \Delta u_{\max }+u_{g}(t+k+1), u_{\max }\right) \\
b_{l}=\max \left(-\Delta u_{\max }+u_{g}(t+k-1),-\Delta u_{\max }+u_{g}(t+k+1), u_{\min }\right) \\
\text { if } k=M-1, \\
b_{u}=\min \left(\Delta u_{\max }+u_{g}(t+k-1), u_{\max }\right) \\
b_{l}=\max \left(-\Delta u_{\max }+u_{g}(t+k-1), u_{\min }\right)
\end{gathered}
$$

The above bounds define the region of $u_{g}(t+k)$ values that will produce a feasible solution. This definition is followed by the generation of a random binary number, $b_{r} . u_{g}(t+k)$ is modified by the following equations based on the value of $b_{r}$ :

$$
\begin{aligned}
& u_{g}(t+k)=u_{g}(t+k) \\
& +\left(b_{u}-u_{g}(t+k)\right)\left(1-r^{\left(1{\text {-iter } / \text { iter }_{\max }}\right)}\right) \text { if } b_{r}=0, \\
& u_{g}(t+k)=u_{g}(t+k) \\
& \left.-\left(u_{g}(t+k)-b_{l}\right)\left(1-r^{\left(1-\text { iter }_{\text {iter }} \max \right.}\right)\right) \text { if } b_{r}=1,
\end{aligned}
$$

where $r$ is a random number between 0 and 1 , iter is the number of iterations performed so far and iter $r_{\max }$ is the expected final number of iterations. 
Step 6 (repeat or stop): If the maximum allowed time has not expired, set iter $=$ iter +1 and return the algorithm to Step 2. Otherwise stop the algorithm and select the chromosome that produces the lowest objective function throughout the entire procedure.

The above simplified genetic algorithm makes it possible to calculate the optimal control in real time.

\section{APPLICATION TO NUCLEAR REACTOR POWER CONTROL}

Fig. 4 shows a schematic block diagram of the model predictive controller. The block "Optimization by GA" calculates the optimal control inputs of the R5 control rod position and the part-strength control rod position minimizing the cost function of Eq. (1) using the genetic algorithm described in subsection 3.2. The block "Output prediction by an SVR model" predicts the future outputs of the power level and the ASI by using the SVR model.

In this study, the developed power and ASI controller was applied to a 3-dimentional reactor analysis code, the Multipurpose Analyzer for Static and Transient Effects of Reactor (MASTER) [20]. The MASTER code developed by KAERI is a nuclear analysis and design code that can simulate the Pressurized Water Reactor (PWR) and Boiling Water Reactor (BWR) cores in 3-dimensional geometry. This code was designed to have a range of capabilities, such as a static nuclear reactor core design, transient nuclear reactor core analysis and operation support. The MASTER code was written in FORTRAN and the proposed model predictive control algorithm was implemented in MATLAB [21].

Initially, the reactor power and ASI controller was designed using the model predictive control optimized by a genetic algorithm and applied to an APR+ nuclear plant modeled using the MASTER code. The thermal power is regulated by five regulating control rod banks as well as by changing the concentration of boric acid, which absorbs neutrons strongly, in the reactor coolant. In this study, it was assumed that the power level and ASI are controlled by regulating control rod banks, R5, R4, R3, $\mathrm{R} 2$ and $\mathrm{R} 1$ and part-strength control rods $\mathrm{P} 1$ and $\mathrm{P} 2$. A leading insertion bank of the regulating control banks is $\mathrm{R} 5$. The regulating control rod banks are inserted in the order of R5, R4, R3, R2 and R1 by being overlapped $(228.6 \mathrm{~cm})$ with each other (refer to Fig. 5), and these regulating banks are withdrawn in opposite order in the case of withdrawal. For example, if they are inserted from the top position $(381 \mathrm{~cm})$, when the R5 control rod bank is inserted first and approaches the $152.4 \mathrm{~cm}(=381$ 228.6 in case of a $228.6 \mathrm{~cm}$ overlap) axial position, the $\mathrm{R} 4$ control rod bank goes into the reactor core together

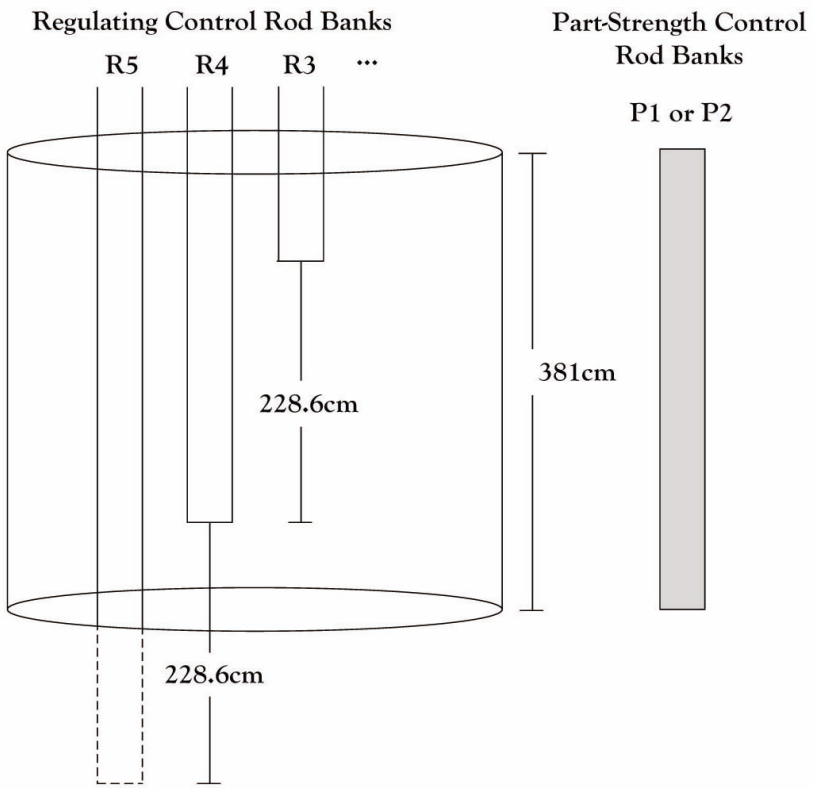

Fig. 5. Overlapped Positions of the Regulating Control Rod Banks.

Model Predictive Control

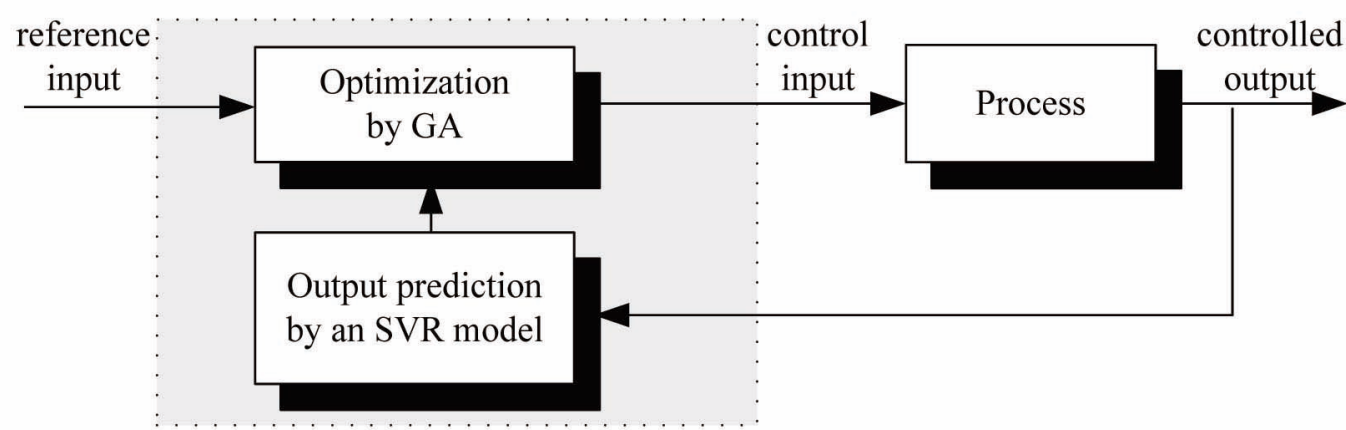

Fig. 4. Schematic Block Diagram of the Proposed Model Predictive Controller. 

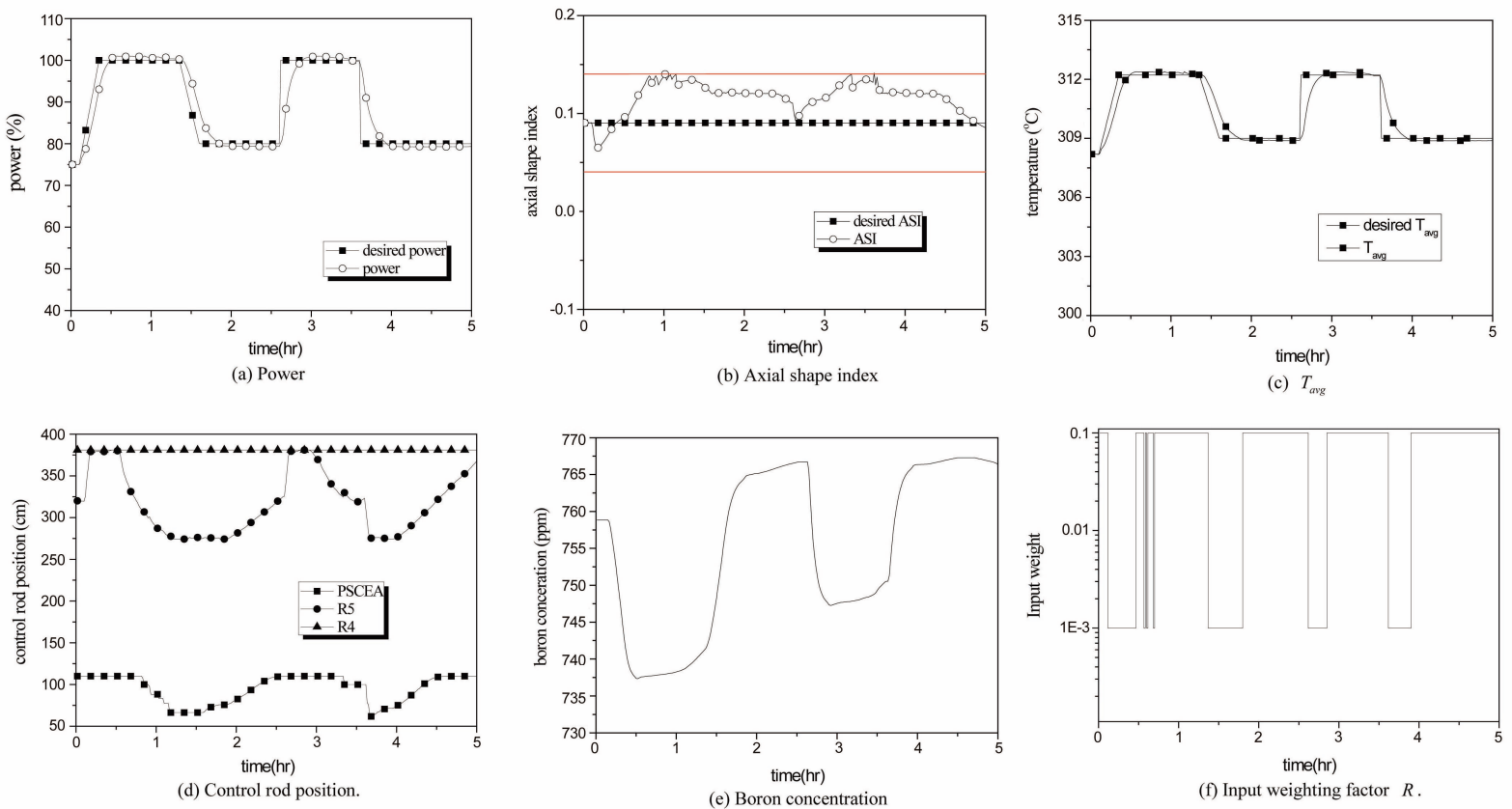

Fig. 6. Performance of the Rapid Change Using the Proposed Model Predictive Controller.

with the R5 control rod bank. As shown in Fig. 5, although all the control rods cannot go down below the bottom of the reactor core, the positions of all the regulating control rod banks can be described by the pseudo position of regulating control rod bank R5, which is a control input and fictitiously goes down to the bottom of the reactor core. Actually, the control rod cannot be inserted below the bottom of the reactor core. That is, it cannot be negative when the position of the control rod is measured from the bottom of a reactor core. On the other hand, all other bank positions can be expressed by the position of the first inserted control rod bank R5 using the negative fictitious position of control rod bank R5 and the specified overlap between the sequential banks. For example, if the pseudoposition of the control rod bank R5 is $-300 \mathrm{~cm}$ and the overlap is $228.6 \mathrm{~cm}$, the actual positions of the control rod banks R5, R4, R3, R2, and R1 is $0 \mathrm{~cm}$ (which is not $-300 \mathrm{~cm}$ ), $0 \mathrm{~cm}$ (which is not -71.4), $157.2 \mathrm{~cm}, 381 \mathrm{~cm}$ (which is not $385.8 \mathrm{~cm}$ ), and $381 \mathrm{~cm}$ (which is not 614.4 $\mathrm{cm}$ ), respectively (refer to Fig. 5 and note that the top position is $381 \mathrm{~cm}$ ).

The model predictive controller for the power level and ASI control is subject to the following constraints:

$$
\begin{aligned}
& \Delta u(t+k-1)=0 \text { for } k>M, \\
& -914.4 \mathrm{~cm} \leq u(t) \leq 381 \mathrm{~cm}, \\
& -1.27 \times T \leq \Delta u(t) \leq 1.27 \times T,
\end{aligned}
$$

where $T$ is a sampling time of $5 \mathrm{sec}$. The pseudo-position of the $\mathrm{R} 5$ regulating control bank, which is a control input, is higher than the four overlap lengths $(-914.4 \mathrm{~cm})$ below the bottom of reactor core and lower than the top position of the reactor core (refer to Fig. 5). The maximum speed of the regulating control rods is 30 inches $/ \mathrm{min}(1.27 \mathrm{~cm} / \mathrm{sec})$. The optimal control input can be obtained by solving the minimization objective function of Eq. (1) using a genetic algorithm. Most of the computation time is invested in calculation of the reactor dynamics and that of the controller is insignificant. The sampling time was 5 sec. Figs. 6-7 show the simulation results of the rapid change and daily load-follow.

Figs. 6-7 show the simulation results. The desired power of the rapid change operation in Fig. 6 was initially $75 \%$. It increased steadily to $100 \%$ from $0.1 \mathrm{hr}$ and then decreased gradually to $80 \%$ from $1.3 \mathrm{hr}$. In addition, it increased slowly from $80 \%$ to $100 \%$ at $2.6 \mathrm{hr}$ and decreased in stages from $100 \%$ to $80 \%$ at $3.6 \mathrm{hr}$. The load-follow operation in Fig. 7 was initially $75 \%$, decreased gradually to $50 \%$ from $0.1 \mathrm{hr}$ and increased steadily to $100 \%$ from $7.1 \mathrm{hr}$. In addition, it decreased from $100 \%$ to $50 \%$ insteps at $23.1 \mathrm{hr}$ and increased steadily from $50 \%$ to $100 \%$ at $31.1 \mathrm{hr}$. Figs. 6(a, b) and 7(a,b) show the responses of the nuclear power level and ASI. The power level and ASI follow their desired values quite well. If the ASI error ranges within \pm 0.05 , the controller operates in a single mode. That is, only the power level is controlled. If the ASI error deviates from \pm 0.05 , it is a dual control mode. That is, both the power level and the ASI are controlled. Figs. 6(c) and 7(c) are the average coolant temperature profiles. Figs. 6(d) and 7(d) show the positions of the 

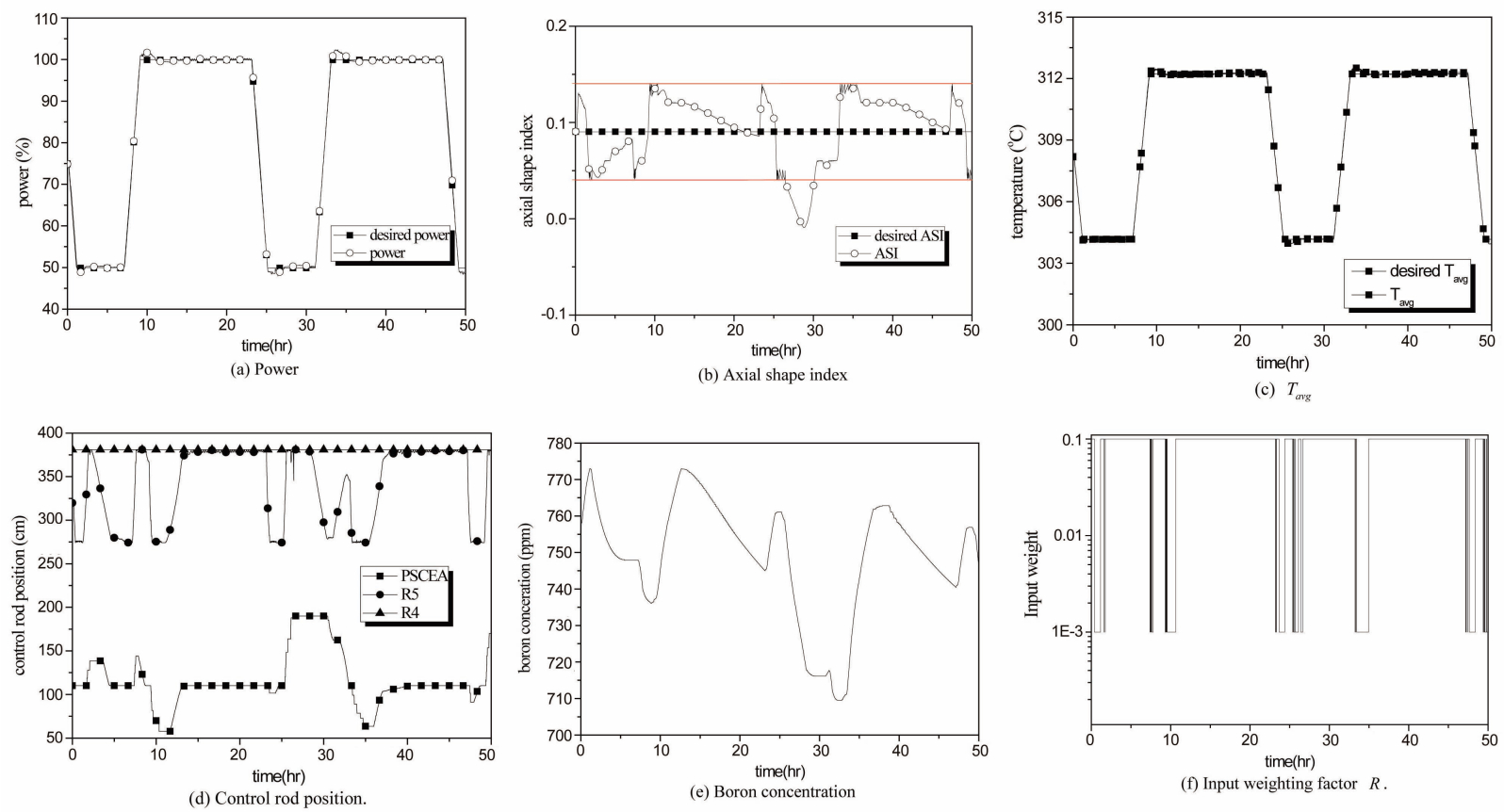

Fig. 7. Performance of the Daily Load-follow Operation Using the Proposed Model Predictive Controller.

regulating control rod banks that describe their overlapped positions well.

Figs. 6(e) and 7(e) show the changes in boron concentration. The boron concentration changes according to the following algorithm:

$$
\begin{aligned}
& \text { if }\left(u_{1}(t)>360 \mathrm{~cm}\right) \text { and }\left(\left(w_{1}(t)-y_{1}(t)\right)>0.2 \%\right) \text { then } \\
& c(t)=c(t-1)-0.001\left(u_{1}(t)-360\right)\left|w_{1}(t)-y_{1}(t)\right| \\
& \text { else if }\left(u_{1}(t)>360 \mathrm{~cm}\right) \text { and }\left(\left(w_{1}(t)-y_{1}(t)\right)<-0.2 \%\right) \text { then } \\
& c(t)=c(t-1)+0.0005\left(u_{1}(t)-360\right)\left|w_{1}(t)-y_{1}(t)\right| \\
& \text { else if }\left(u_{1}(t)<290 \mathrm{~cm}\right) \text { and }\left(\left(w_{1}(t)-y_{1}(t)\right)<-0.2 \%\right) \text { then } \\
& c(t)=c(t-1)+0.001\left(290-u_{1}(t)\right)\left|w_{1}(t)-y_{1}(t)\right| \\
& \text { else if }\left(u_{1}(t)<290 \mathrm{~cm}\right) \text { and }\left(\left(w_{1}(t)-y_{1}(t)\right)>0.2 \%\right) \text { then } \\
& c(t)=c(t-1)-0.0005\left(290-u_{1}(t)\right)\left|w_{1}(t)-y_{1}(t)\right| .
\end{aligned}
$$

In the above algorithm, $c(t)$ is the boron concentration at time $t . u_{1}(t), w_{1}(t)$, and $y_{1}(t)$ are the R5 control rod position, the desired power and the measured power at time $t$. The above algorithm is presented to prevent the R5 control rod position from getting into an uncontrollable region and to maintain the appropriate position of the R5 control rod. Additionally, the boron concentration is adjusted as follows:

$$
\begin{aligned}
& \text { if }\left(u_{1}(t)>\left(2 w_{1}(t)+180\right)\right) \text { then } \\
& c(t)=c(t)-0.03 e^{-3\left|w_{1}(t)-y_{1}(t)\right|} \\
& \text { else if }\left(u_{1}(t)<\left(2 w_{1}(t)+160\right)\right) \text { then } \\
& c(t)=c(t)+0.03 e^{-3\left|w_{1}(t)-y_{1}(t)\right|} .
\end{aligned}
$$

The above algorithm recovers the appropriate boron concentration when the R5 control rod position exists within a specified range according to the desired power level.

The weighting factor $Q$ is normally selected to be unity and the input weighting factor $R$ normally plays an important role in determining the behavior of a closedloop system. Figs. 6(f) and 7(f) show the input weighting factors of the controller responses which varies according to the control mode.

This paper proposed a new reactor power and ASI controller that combines the MPC control methodology, which has received considerable attention as a powerful tool for the control of industrial process systems, with the genetic algorithm and SVR model, which are widely used to solve engineering problems. A simple control algorithm is not so important because these days, digital processors are used in control instead of old hardwired circuits. If the control performance can be improved with a new complex control algorithm, it will be better than a simple control algorithm with low performance. As shown in Figs. 6-7, the proposed controller provides excellent performance, even though the ASI exceeds a set value in a range due to limited control rod capability.

\section{CONCLUSIONS}

The load-following operation of APR + nuclear plants requires the ultimate automatic control and an advanced control method, and was used as a model predictive control 
method for a fast and robust algorithm. In this study, the model predictive controller optimized by a genetic algorithm was developed to control the nuclear power and ASI in APR+. The developed controller was applied to an APR+ nuclear plant, which was modeled using the MASTER code. Based on an SVR model consisting of the control rod position and reactor power, the future reactor power and ASI were predicted using the SVR model. The genetic algorithm is suitable for solving multiple objective functions and can be used to optimize the model predictive controller. The power level and ASI are controlled simultaneously and systematically by regulating the control rod banks and part-strength control rod banks. The proposed controller adjusts the control rod position so that the nuclear reactor power and ASI tracks quite well its change in the setpoint according to the load.

\section{REFERENCES}

[1] W. H. Kwon and A. E. Pearson, "A Modified Quadratic Cost Problem and Feedback stabilization of a Linear System," IEEE Trans. Automatic Control, vol. 22, no. 5, pp. 838-842, 1977.

[2] J. Richalet, A. Rault, J. L. Testud, and J. Papon, "Model Predictive Heuristic Control: Applications to Industrial Processes," Automatica, vol. 14, pp. 413-428, 1978.

[ 3 ] C. E. Garcia, D. M. Prett, and M. Morari, "Model Predictive Control: Theory and Practice - A Survey," Automatica, vol. 25 , no. 3 , pp. $335-348,1989$.

[4] D. W. Clarke, and R. Scattolini, "Constrained Receding Horizon Predictive Control," IEE Proceedings-D, vol. 138, no. 4, pp. 347-354, 1991.

[ 5 ] M. V. Kothare, V. Balakrishnan, and M. Morari, "Robust Constrained Model Predictive Control Using Linear Matrix Inequality," Automatica, vol. 32, no. 10, pp. 1361-1379, 1996.

[6] J. W. Lee, W. H. Kwon, and J. H. Lee, "Receding Horizon $H^{\infty}$ Tracking Control for Time-Varying Discrete Linear Systems,” Intl. J .Control, vol. 68, no. 2, pp. 385-399, 1997.

[7] J. W. Lee, W. H. Kwon, and J. Choi, "On Stability of Constrained Receding Horizon Control with Finite Terminal
Weighting Matrix," Automatica, vol. 34, no. 12, pp. 16071612, 1998.

[ 8 ] M. G. Na, "A Model Predictive Controller for the Water Level of Nuclear Steam Generators," J. Korean Nucl. Soc., vol. 33, no. 1, pp. 102-110, Feb. 2001.

[9] N. Z. Cho and L. M. Grossman, "Optimal Control for Xenon Spatial Oscillations in Load Follow of a Nuclear Reactor," Nucl. Sci. Eng., vol. 83, pp. 136-148, 1983.

[10] P.P. Niar and M. Gopal, "Sensitivity-Reduced Design for a Nuclear Pressurized Water Reactor," IEEE Trans. Nucl. Sci., vol. NS-34, no. 6, pp. 1834-1842, Dec. 1987.

[11] C. Lin, J.-R. Chang, and S.-C. Jenc, "Robust Control of a Boiling Water Reactor," Nucl. Sci. Eng., vol. 102, no. 3, pp. 283-294, July 1989.

[12] M. G. Park and N. Z. Cho, "Nonlinear Model-Based Robust Control of a Nuclear Reactor Using Adaptive PIF Gains and Variable Structure Controller," J. Korean Nucl. Soc., vol. 25, no. 1, pp. 110-124, Mar. 1993.

[13] V. Kecman, Learning and Soft Computing. Cambridge, Massachusetts: MIT Press, 2001.

[14] V.N. Vapnik, Statistical Learning Theory. New York, NY: John Wiley \& Sons, 1998.

[15] V.N. Vapnik, The Nature of Statistical Learning Theory. New York: Springer, 1995.

[16] D. E. Goldberg, Genetic Algorithms in Search, Optimization, and Machine Learning, Addison Wesley, Reading, Massachusetts, 1989.

[17] M. Mitchell, An Introduction to Genetic Algorithms, MIT Press, Cambridge, Massachusetts, 1996.

[18] H. Sarimveis and G. Bafas, "Fuzzy Model Predictive Control of Nonlinear Processes Using Genetic Algorithms," Fuzzy Sets Systems, vol. 139, pp. 59-80, 2003.

[19] Man Gyun Na and In Joon Hwang, "Design of a PWR Power Controller Using Model Predictive Control Optimized by a Genetic Algorithm," Nucl. Eng. Tech., vol. 38, no. 1, pp. 81-92, Feb. 2006.

[20] B. O. Cho, H. G. Joo, J. Y. Cho and S. Q. Zee.: MASTER: Reactor Core Design and Analysis Code, Proc. 2002 Int. Conf. New Frontiers of Nuclear Technology: Reactor Physics (PHYSOR 2002), Seoul, Korea, Oct. 7-10, 2002.

[21] Math Works, MATLAB 2011, The Math Works, Natick, Massachusetts, 2011. 